\title{
Tratamiento con GH en niños con talla baja sin deficit de esta hormona
}

Effect of growth hormone treatment on adult height of children with idiopathic short stature.

Hintz R, Attie K, Baptista J, Roche A, for the Gennentech Collaborative Group. N Engl J Med 1999; 340: 507-7

\section{Objetivo}

Evaluar el efecto sobre la talla adulta del tratamiento prolongado con hormona de crecimiento $(\mathrm{GH})$ en niños con talla baja idiopática sin déficit de hormona.

\section{Diseño}

Ensayo clínico no randomizado. Se evaluó la talla adulta alcanzada por los niños tratados con $\mathrm{GH}$.

\section{Lugar}

Estudio multicéntrico realizado en 14 centros de Estados Unidos.

\section{Pacientes}

Entraron en el estudio 121 pacientes y sólo se analizaron los datos de los 80 que alcanzaron la talla adulta. Todos eran prepúberes y tenían una talla debajo del percentilo 3, una velocidad de crecimiento para su edad inferior al percentilo 50 y un nivel máximo de $\mathrm{GH}$ post estímulo de, al menos, 10ug/L.

\section{Intervención}

Los 121 niños recibieron $\mathrm{GH}$ a una dosis de $0.3 \mathrm{mg} / \mathrm{kg} /$ semana. Un total de 80 pacientes completaron dos a diez años de tratamiento y alcanzaron la talla adulta.

Fuente de financiamiento: Genentech y subsidios del Public Health Service.

\section{Medición de los resultados principales}

Se evaluó la talla final alcanzada y se la comparó con la talla adulta predicha al inicio del tratamiento según la información correspondiente a grupos históricos no tratados.

\section{Resultados principales}

De los 121 niños que iniciaron el estudio sólo pudo evaluarse a los 80 que lo completaron. La talla media adulta de los 80 niños tratados fue mayor que la talla adulta predicha al inicio del tratamiento, no alcanzando la talla blanco media. Sin embargo las respuestas individuales fueron muy variables y 63 pacientes (79\%) alcanzaron una talla adulta mayor que la predicha. La diferencia media entre la talla alcanzada y la predicha fue de $5+5.1 \mathrm{~cm}$ para los varones y de $5.9+5.2 \mathrm{~cm}$ para mujeres; habiéndose registrado una diferencia mayor a $5 \mathrm{~cm}$ entre la talla adulta y la talla predicha en el $51 \%$ de los varones tratados y en el $61 \%$ de las mujeres.

Comparando con los resultados en los niños con baja talla idiopática no tratados, el aumento medio en la talla adulta de los niños tratados fué $9.2 \mathrm{~cm}$ para los varones y de $5.7 \mathrm{~cm}$ para las mujeres.

\section{Conclusiones}

La administración prolongada de GH puede aumentar la talla adulta por encima de la talla adulta inicialmente predicha y por encima de la talla adulta alcanzada por los niños de controles históricos no tratados.

\section{COMENTARIO}

Este estudio trata de demostrar que la utilización de GH mejora la talla adulta de los niños con talla baja idiopática. De los 80 niños evaluados, el 53\% alcanzó una talla adulta mayor a $5 \mathrm{~cm}$ respecto de la talla predicha inicialmente. Sin embargo, 31\% de los 121 niños iniciales no continuaron el tratamiento. ¿Será que la talla baja no alcanza a ser un estímulo suficiente para aceptar este tratamiento? ¿Es una respuesta de 53\% (o sólo de 35\%) lo suficientemente buena como para justificar el empleo de un tratamiento crónico, costoso y por via inyectable a niños, en principio, normales? En general, un paciente con talla baja tiene antecedentes familiares de talla baja o de retardo puberal. Se ha observado que estos niños pueden, espontáneamente, aumentar su talla final en más de un desvío estándar con respecto a la talla de la primera consulta; mientras que en aquellos con retardo puberal (edad ósea atrasada) este aumento puede ser de más de dos desvios estándar ${ }^{1}$. Por otro lado hay que tener en cuenta que el error en la predicción de la talla adulta de un niño bajo no es despreciable (estudios que han utilizado controles históricos o no randomizados sobreestiman un $30 \%$ los beneficios del tratamiento 2); por lo tanto, ¿cómo evaluar que el tratamiento es justificable?.

La velocidad de crecimiento es un parámetro muy importante a evaluar. Si ésta está por debajo del percentilo 25 y la talla se aleja cada vez más de la media normal se justifica medir la GH. Si el niño crece normalmente y pre-

\section{Dra. Titania Pasqualini}

Sección de Endocrinología Pediátrica. Departamento de Pediatría. Hospital Italiano de Buenos Aires.

\section{Referencias}

1. Price DA. 1996. Spontaneous adult height in patients with idiopathic short stature. Horm Res 45 (Suppl 2):59-63.

2. Taback SP, Guyda HJ Van Vliet G. 1999. Pharmacological manipulation of height: qualitative review of study populations and design. Clin Invest Med 22: 53-59.

3. American Academy of Pediatrics Committee on Drugs and Committee on Bioethics: Considerations related to the use of recombinant human growth hormone in children: 1997 . Pediatrics $99: 122-129$.

4. Ranke MB, Price DA, Albertsson-Wikland K, Maes, Lindberg A. 1997. Factors determining pubertal growth and final height in growth hormone treatment of idiopathic growth hormone deficiency. Analysis of 195 patients of the Kabi Pharmacia International Growth Study. Horm Res 48: 62-71.

5. Blethen SL, Baptista J, Kuntze J, Foley T, LaFreanchi S, Johanson A.1997. Adult height in GH-deficient children treated with biosynthetic growth hormone. J Clin Endocrinol. Metab 82: 418-420. 6. Cuttfield W, Lindberg A, Albertsson-Wikland K, Chatelain P, Ranke MB, Wilton P. 1999. Final height in idiopathic growth hormone deficiency. Acta Paediatrica 88 (Suppl): $72-75$.

7. Rosenfeld RG, Attie KM, France J, et al. 1998. Growth hormone therapy in Turner syndrome: Beneficuial effect on adult height. J Pediatr 132: 319-313.

8. Haeusler G, Schmitt K, Blumel P, Plochl E, Waldhor TH, Frisch H. 1996 Growth hormone in combination with anabolic steroids in patients with Turner syndrome: effect on bone maduration and final height. Acta Paediatr 85: 1408-1414.

9. Sas TCJ, De Muinck Keizer-Schrama SMPF, Stijnen T, Jansen M, Otten BJ, Hoorweg-Nijman JJG, Vulsma T, Massa GG, Rouwé CW, Reeser HM, Gerver WJ, Gosen JJ, Rongen-Westerlaken C, Drop SLS. 1999. Normalization of height in girls with Tumer syndrome after long-term growth hormone treatment: Results of a randomized dose-response trial. J Clin Endocrinol Metab 84: $4607-4612$.

10. Guyda HJ. 1996. Growth hormone treatment of non growth hormone deficicient subjects: The International Task Force (TIF) Report. Clin Pediatr Endocrinol 5: 11-18.

11. Buchlis JG, Irizarry L, Crotzer BC, Shine BJ, Allen L, MacGillivray MH. 1998. Comparison of final heights of growth hormone-treated vs. untreated children with idiopathic growth failure. J Clin Endocrinol Metab 83: 1075-1079.

12. Rekers-Mombarg LTM, Wit J-M, Massa GG,Et al. 1996. Spontaneous grwowth in idiopathic short stature. Arch Dis Child 75: 175-180. 